\title{
SEGREGAÇÃO DE FERTILIZANTES APLICADOS A LANÇO
}

\author{
JOSÉ P. MOLIN ${ }^{1}$, THIAGO M. MACHADO ${ }^{2}$, RAPHAEL P. MAGALHÃES ${ }^{3}$, \\ GUSTAVO D. C. FAULIN ${ }^{4}$
}

\begin{abstract}
RESUMO: A uniformidade transversal da distribuição de fertilizantes e corretivos a lanço tem grande dependência da qualidade e da condição física do produto a ser aplicado. Especialmente no caso das formulações, esse aspecto é relevante e por isso o presente trabalho teve o objetivo de verificar a distribuição dos grãos e a constância na formulação aplicada na extensão da largura de trabalho de uma máquina distribuidora a lanço com dois fertilizantes. Foram efetuadas análises químicas do produto depositado em coletores dispostos transversalmente e analisadas as distribuições individuais dos elementos das fórmulas. Os resultados evidenciam que os fertilizantes apresentaram diferenças na sua formulação ao longo da largura, sendo que o fertilizante que continha micronutrientes obteve maiores desvios (de até 100\%) em relação à formulação aplicada, e sua segregação foi elevada em relação aos macronutrientes primários (até 9,5\% para $\mathrm{N}$ ) e secundários estudados (até 39,0\% para Ca), o que também indica distribuição de pior qualidade dos fertilizantes. O outro fertilizante apresentou desvios de até $17,8 \%$ para $\mathrm{N}$ e $34,1 \%$ para $\mathrm{K}$ na formulação ao longo da largura de aplicação.
\end{abstract}

PALAVRAS-CHAVE: aplicação a lanço, deposição transversal, adubadora.

\section{SPREADING SEGREGATION OF TWO FERTILIZERS}

ABSTRACT: The transversal distribution uniformity of spreaders frequently used for fertilizer and lime distribution are dependent of the quality and physical condition of the product to be distributed. Especially for formulated fertilizers it is relevant and for this reason the objective of this work was verifying the distribution of particles and constancy on the applied composition throughout the spreading width of a spread machine with two fertilizers. The fertilizer collected along the distribution was submitted to chemical analyses and the distribution of each individual element was analyzed. The results indicate that both fertilizers showed differences on the formulation considering the distance from the throwing position to where they were collected. The composition which had micronutrients had the larger degradation to the applied formula (up to $100 \%$ ) on them and elevated segregation on macronutrients (up to $9.5 \%$ for $\mathrm{N}$ ) and secondary nutrients (up to $39.0 \%$ for $\mathrm{Ca}$ ), showing a bad distribution for them. The other fertilizer also presented deviations of up to $17.8 \%$ for $\mathrm{N}$ and $34.1 \%$ for $\mathrm{K}$ on the fertilizer formula along the transversal distribution.

KEYWORDS: fertilizer spreading, transversal deposition, fertilizer segregation.

\section{INTRODUÇÃO}

No Brasil, a tendência da utilização de adubação distribuída a lanço é crescente, e as máquinas comumente utilizadas têm como característica principal grande dependência em relação à qualidade e à condição física do produto a ser aplicado. Para a qualificação da sua deposição, é necessária a análise da regularidade da distribuição transversal, que, de acordo com as normas ISO 5690/1 (1985) e ASAE S341.2 (1995), pode ser efetuada baseando-se no coeficiente de variação (C.V.) da distribuição dos materiais empregados.

\footnotetext{
${ }^{1}$ Eng ${ }^{\circ}$ Agrícola, Livre Docente, Departamento de Engenharia Rural, ESALQ/USP, Piracicaba - SP, Fone: (0XX19) 3447.8502, jpmolin@esalq.usp.br

${ }^{2}$ Eng $^{\circ}$ Agrícola, Doutorando, FCA/UNESP, Botucatu - SP.

${ }^{3}$ Eng ${ }^{0}$ Agrônomo, MSc, Campinas - SP.

${ }^{4}$ Eng ${ }^{\circ}$ Agrônomo, Doutorando, ESALQ/USP, Piracicaba - SP.

Recebido pelo Conselho Editorial em: 10-3-2008
}

Aprovado pelo Conselho Editorial em: 3-10-2009 
Para a aplicação de fertilizantes minerais, utiliza-se normalmente a mistura de grãos que, segundo MALAVOLTA et al. (2000), é aquela em que os nutrientes se encontram em diferentes grânulos. Segundo ALCARDE et al. (1989), as características de qualidade dos fertilizantes minerais podem ser classificadas como de natureza química, física e físico-química. As características físicas, basicamente, definem a qualidade de distribuição dos fertilizantes. Essas são mais difíceis de serem controladas pela legislação oficial e podem ser subdivididas em granulometria, consistência e fluidez. A fluidez relaciona-se com o ângulo de repouso dos fertilizantes, a qual irá influenciar na exatidão e na regularidade da dosagem do produto. A análise granulométrica pode ser determinada à luz da norma ISO 5690/1 (1985), que preconiza a agitação de um quilograma do produto através de peneiras dispostas em ordem decrescente de tamanho de malha, apresentando o resultado desse peneiramento em números percentuais de massas retidas em cada classe.

Em decorrência da composição granulométrica, os fertilizantes sólidos podem apresentar segregação, que é a separação das partículas componentes de uma mistura por ordem de tamanho e densidade, sendo essa potencializada pela desuniformidade do produto (POPP \& ULRICCH, 1985). As partículas de fertilizantes também podem ser desuniformes em decorrência da qualidade do processo industrial e da quebra de grânulos devido à baixa consistência. No entanto, SMITH (1960) afirma que a segregação prejudica particularmente a uniformidade dos teores de micronutrientes quando uma quantidade relativamente pequena da fonte, geralmente em formato de pó, é adicionada às misturas de grânulos.

A particularidade da segregação é um tema pouco abordado no Brasil, tanto na pesquisa relacionada à qualidade de adubos como no estudo relativo às máquinas adubadoras. $\mathrm{O}$ presente estudo teve o objetivo de verificar a distribuição dos grãos e a constância na formulação aplicada ao longo da largura de trabalho de uma máquina distribuidora a lanço com dois fertilizantes, uma vez que, assim como existem variações quantitativas nessa distribuição, pode-se supor que, qualitativamente, diferenças ocorram em função da segregação de cada nutriente granulado, devido a densidades distintas apresentadas entre eles.

\section{MATERIAL E MÉTODOS}

Foi utilizada uma distribuidora de fertilizantes a lanço (Tornado 1.300 Geração III, Stara Sfil S.A.), montada em um trator modelo MF 292 e acionada pela TDP. A máquina possui mecanismo dosador gravitacional, com distribuidor centrífugo formado por dois rotores (discos) horizontais em aço inox e um depósito com capacidade volumétrica de $1,3 \mathrm{~m}^{3}$.

Dois fertilizantes comerciais compostos pela mistura de grânulos mostrados na Tabela 1 foram utilizados nos ensaios, sendo um com micronutrientes $(\mathrm{X})$ e o outro desprovido desses (Y).

TABELA 1. Percentagem de cada nutriente na formulação, declarada pelo fabricante dos dois fertilizantes utilizados nos ensaios. Nutrients content on the fertilizer formula, as stated by the manufacturer for both fertilizers used on the tests.

\begin{tabular}{|c|c|c|c|c|c|c|c|c|}
\hline \multirow{2}{*}{ Denominação } & $\mathrm{N}$ & $\mathrm{P}_{2} \mathrm{O}_{5}$ & $\mathrm{~K}_{2} \mathrm{O}$ & $\mathrm{Ca}$ & $\mathrm{S}$ & $\mathrm{B}$ & $\mathrm{Zn}$ & Mo \\
\hline & \multicolumn{8}{|c|}{$\%$} \\
\hline$X$ & 6 & 18 & 18 & 5 & 5 & 0,04 & 0,1 & 0,004 \\
\hline $\mathrm{Y}$ & 6 & 18 & 18 & 5 & 6 & - & - & - \\
\hline
\end{tabular}

Os fertilizantes foram avaliados quanto à análise granulométrica, umidade e ângulo de repouso. Para a determinação da granulometria, foram utilizadas sete peneiras, com aberturas de $4.760 ; 2.380 ; 2.000 ; 1.000 ; 0.500 ; 0.250$ e $0.105 \mathrm{~mm}$. O peneiramento do fertilizante foi efetuado durante 5 minutos com as peneiras dispostas em ordem decrescente, empregando-se três repetições por fertilizante. A análise da umidade dos produtos foi efetuada pelo método gravimétrico, pesandose seis amostras de aproximadamente $100 \mathrm{~g}$, antes e após a secagem em estufa, a $105^{\circ} \mathrm{C}$, por $48 \mathrm{~h}$. 
O ângulo de repouso foi calculado, utilizando-se da mesma metodologia proposta por MOHSENIN (1986), que propõe a utilização de dispositivo retangular que se adapta a um funil e a uma plataforma circular com altura regulável. Após o escoamento do produto pelo referido funil, calcula-se o ângulo desse produto em repouso.

Para o ensaio da vazão do mecanismo dosador da máquina, utilizaram-se de quatro posições $(2 ; 4 ; 6$ e 8) do indicador do dispositivo de ajuste de aberturas do mecanismo dosador. A rotação de trabalho na TDP foi verificada com o auxílio de um tacômetro para que se mantivessem as $540 \mathrm{rpm}$, segundo recomendação do fabricante da máquina. Foi coletado o fertilizante em cada posição do mecanismo dosador por 30 segundos. Nessa etapa, o material foi pesado utilizando-se de uma célula de carga da marca KYOWA, com capacidade para $5.000 \mathrm{~N}$, e um indicador de célula de carga da marca MICRO P. Após a obtenção da curva de vazão para ambos os fertilizantes, definiu-se a abertura do mecanismo dosador correspondente à dosagem de $250 \mathrm{~kg} \mathrm{ha}^{-1}$ para ambos.

Para a avaliação da largura efetiva de aplicação da máquina, foram seguidos os procedimentos da norma ISO 5690/1 (1985), que propõe a utilização de coletores padronizados com $1.000 \mathrm{~mm}$ de comprimento e $250 \mathrm{~mm}$ de largura, com profundidade máxima de $150 \mathrm{~mm}$, prevendo, também, a utilização de um dispositivo para evitar o ricochete do produto, utilizado conforme sugerido por MOLIN \& MAZOTTI (2000). O procedimento foi efetuado com condições de velocidade do vento inferiores a 2,0 $\mathrm{m} \mathrm{s}^{-1}$, utilizando-se, para o seu monitoramento, de anemômetro MDA-10 (MINIPA). Esse monitoramento foi efetuado no momento da passagem da máquina sobre os coletores.

A altura da máquina e demais ajustes foram efetuados conforme o manual do usuário. Foram utilizados coletores numerados de 1 a 100, dispostos lado a lado, na perpendicular em relação à trajetória do trator, que foi efetuada sempre no mesmo sentido. Iniciou-se o trajeto com uma distância suficiente para a estabilização do conjunto antes de cruzar a linha dos coletores. Havia espaços no centro da linha dos coletores destinados à passagem do trator, equivalentes a três coletores $(750 \mathrm{~mm})$ em cada rodado.

Com a dose estipulada de $250 \mathrm{~kg} \mathrm{ha}^{-1}$, foram executadas três passadas da máquina na velocidade de $2,2 \mathrm{~m} \mathrm{~s}^{-1}\left(8 \mathrm{~km} \mathrm{~h}^{-1}\right)$, as quais acumularam material suficiente para a análise da distribuição transversal de produto. A largura efetiva da máquina foi obtida, utilizando-se do programa Adulanço (GONÇALVES et al., 2003), que define a largura efetiva como função da uniformidade de distribuição do produto. Nesse caso, utilizou-se da máxima largura com C.V. da distribuição transversal de $15 \%$. Tal uniformidade foi determinada a partir da massa de produto, mensurada por balança de precisão GEHAKA, com acurácia de $9,8 \times 10^{-5} \mathrm{~N}$, do material depositado em cada coletor.

Para a análise da segregação de grânulos, utilizou-se do produto tomado em um coletor para cada metro de largura para a análise laboratorial de conteúdo de componentes. Para as amostras com quantidades não suficientes para a análise química (em torno de 2,0 g), foram individualizados os grânulos e separados visualmente por critério de cor, após a pesagem. O elemento Mo foi desconsiderado das análises em função de seu baixo teor.

Para as análises de segregação dos nutrientes contidos nos fertilizantes, utilizou-se do mesmo método da determinação da largura efetiva da máquina, estabelecendo a largura ótima de trabalho equivalente à maior largura com C.V. de $15 \%$, considerando-se separadamente a deposição de cada nutriente. Quantificou-se, também, a fórmula depositada nos extremos e no centro da faixa de aplicação, considerando a largura efetiva da máquina, e avaliou-se o quanto o valor obtido diferia da fórmula utilizada. Com o auxílio da estatística descritiva, analisando os fatores de posição e de variação, comparou-se o resultado de ambos os fertilizantes e em percurso contínuo (fechando ou abrindo quadro) e alternado (em "vai e vem") com manobra nas cabeceiras do talhão. No circuito contínuo, existe a sobreposição do lado direito com o lado esquerdo da deposição da máquina, e vice-versa, minimizando possíveis desequilíbrios causados pela assimetria de deposição da máquina. Já no circuito alternado, lados iguais se sobrepõem (alternado direito e alternado esquerdo), somando eventuais assimetrias na distribuição do produto. 


\section{RESULTADOS E DISCUSSÃO}

$\mathrm{O}$ ângulo de repouso do fertilizante $\mathrm{X}$ foi de $31,2^{\circ}$ e para o fertilizante $\mathrm{Y} 33,1^{\circ}$. Os fertilizantes X e Y apresentaram, respectivamente, umidades de 2,9\% e 1,3\%. A síntese da análise granulométrica dos fertilizantes é apresentada na Figura 1. Observou-se concentração em torno de $70 \%$ de partículas com diâmetro acima de $2,38 \mathrm{~mm}$ e o restante com diâmetro entre 0,50 e 2,38 mm, para ambos os produtos.

A partir da calibração da vazão do mecanismo dosador, determinou-se o ponto de regulagem para o fertilizante a ser aplicado, optando-se pela posição da alavanca de abertura 7,5, referente à dosagem de $250 \mathrm{~kg} \mathrm{ha}^{-1}$. O histograma da distribuição transversal de ambos os fertilizantes para a dosagem estabelecida pode ser observado na Figura 2.

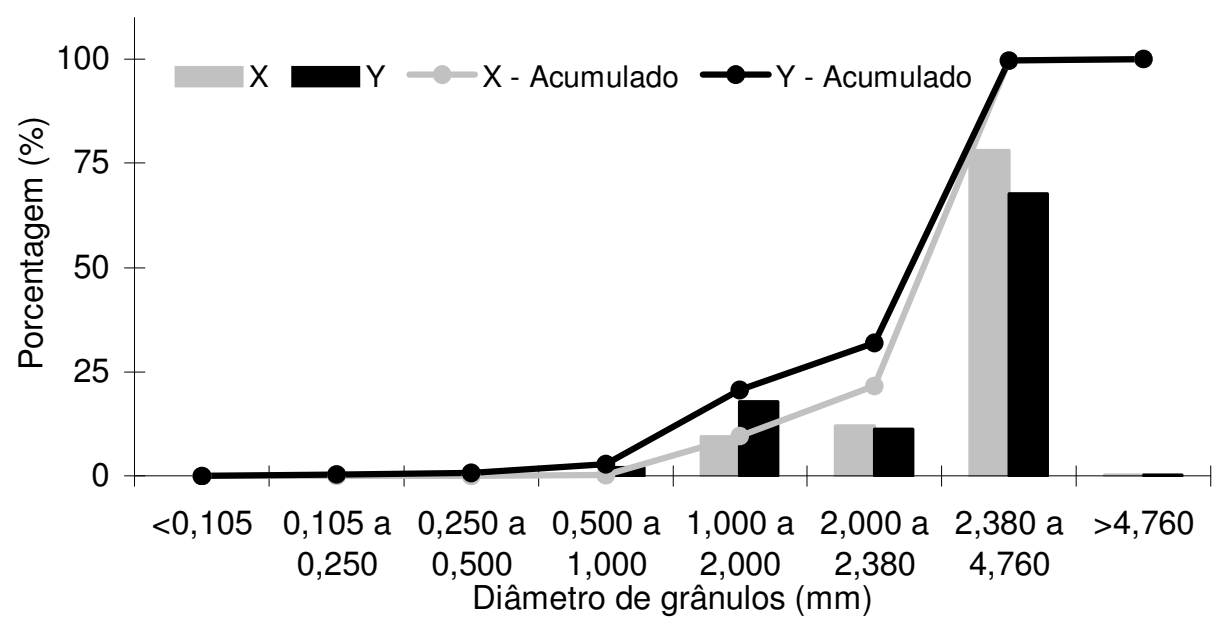

FIGURA 1. Percentagem de material retido e valor acumulado para cada classe (peneira) de tamanho de partículas dos fertilizantes analisados. Percentage of material retained on each class (sieve) of particles size for the fertilizers analyzed.
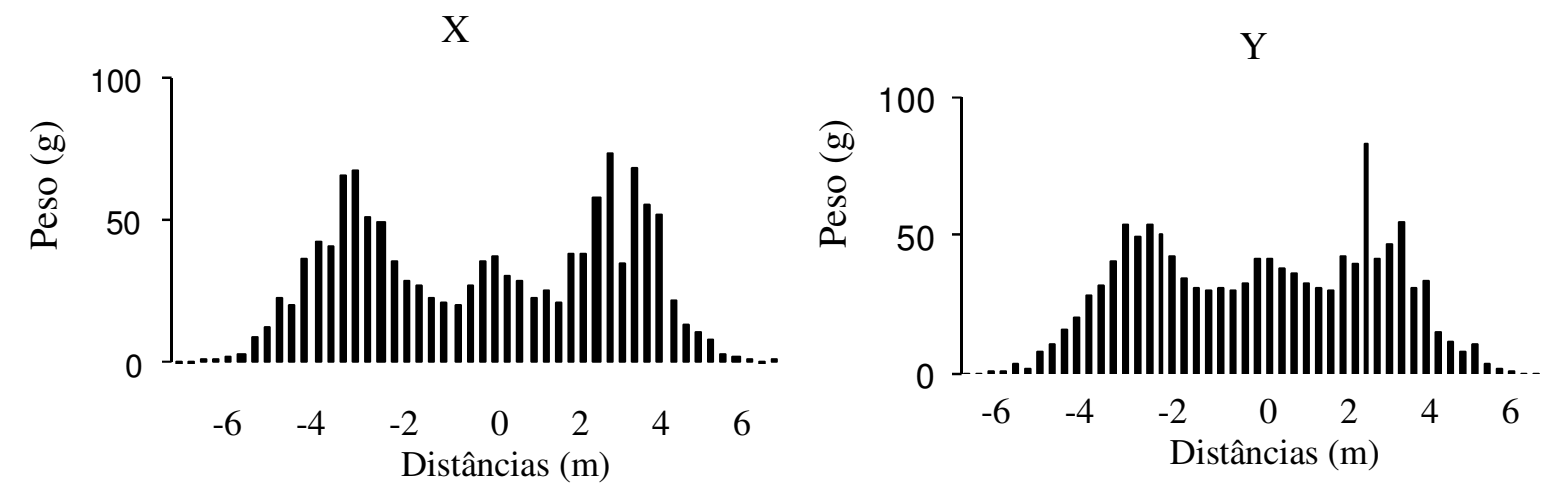

FIGURA 2. Distribuição transversal do fertilizante $X$ (esquerda) e do fertilizante $Y$ (direita), acumulado em cada coletor, referente à dosagem de $250 \mathrm{~kg} \mathrm{ha}^{-1}$. Transversal distribution for fertilizer $\mathrm{X}$ (left) and fertilizer $\mathrm{Y}$ (right), accumulated on each collector, for the application rate of $250 \mathrm{~kg} \mathrm{ha}^{-1}$.

A geometria da distribuição dos fertilizantes apresentou formato em "W", com picos de maior quantidade de fertilizante aplicado nas laterais e leve ascensão no centro. A relação entre o C.V. da distribuição do produto aplicado e a largura útil de aplicação em diferentes percursos para a dose utilizada é ilustrada na Figura 3, relacionada, respectivamente, aos fertilizantes X e Y. 
Com base nas curvas do C.V., como função da largura efetiva de trabalho, e considerando um limite máximo de $15 \%$ para esse valor como aceitável para a distribuição do produto no campo, constatou-se que as larguras de trabalho máximas são aquelas apresentadas na Tabela 2. Observa-se que as larguras de trabalho são consistentes e variam menos para o fertilizante $X$, entre 7,5 e 8,0 m, e entre 7,3 e 8,2 m, para o fertilizante Y.

As larguras efetivas da faixa de aplicação, calculadas levando em consideração cada nutriente, para o C.V. de 15\%, estão apresentadas na Tabela 3. Observa-se que as larguras efetivas alteraram sensivelmente para alguns dos elementos da fórmula dos fertilizantes. É o caso do elemento $\mathrm{K}_{2} \mathrm{O}$ do fertilizante $\mathrm{Y}$, que teve largura efetiva variando de 4,3 a 7,4 m, e no fertilizante $\mathrm{X}$, variando de 4,6 a $7,9 \mathrm{~m}$. Os micronutrientes presentes no fertilizante $\mathrm{X}$ foram ainda mais sensíveis, como o elemento $\mathrm{Zn}$, que variou de 4,4 a $8,4 \mathrm{~m}$ de largura efetiva.
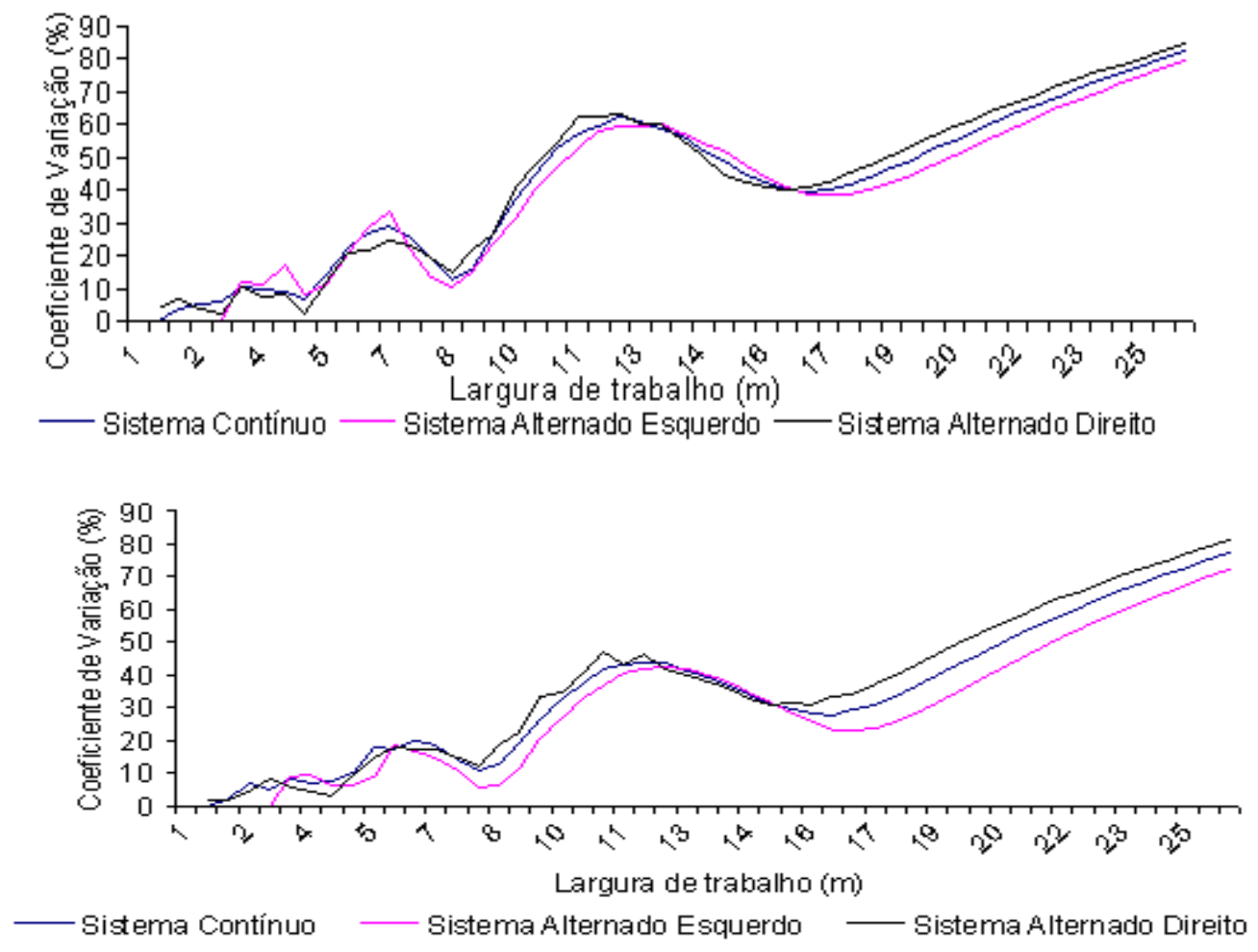

FIGURA 3. Relação entre os valores de C.V. da distribuição transversal e a largura de aplicação para o fertilizante X (superior) e Y (inferior). Relationship between C.V. values for the transversal distribution and the application width for fertilizer $X$ (top) and fertilizer Y (bottom).

TABELA 2. Largura de trabalho efetiva da máquina para o C.V. da distribuição transversal de $15 \%$ nas três possíveis combinações, resultantes do método de percurso da máquina no campo. Machine effective working width for a $15 \%$ transversal distribution C.V. for the three possible field tracks.

\begin{tabular}{cccc}
\hline \multirow{2}{*}{ Fertilizantes } & \multicolumn{3}{c}{ Largura de Trabalho $(\mathrm{m})$ para o Percurso } \\
\cline { 2 - 4 } & \multirow{2}{*}{ Contínuo } & Alternado \\
\cline { 3 - 4 } & & Esquerdo & Direito \\
\hline $\mathrm{X}$ & 7,9 & 8,0 & 7,5 \\
$\mathrm{Y}$ & 7,7 & 8,2 & 7,3 \\
\hline
\end{tabular}


TABELA 3. Larguras efetivas de aplicação, considerando cada nutriente individualmente, para os dois fertilizantes analisados e utilizando C.V. de $15 \%$ na regularidade de distribuição transversal. Application working width considering each nutrient individually for the two fertilizers analyzed and using a C.V. of $15 \%$ for the transversal regularity.

\begin{tabular}{|c|c|c|c|c|c|c|}
\hline \multirow{5}{*}{ Nutrientes } & \multicolumn{6}{|c|}{ Fertilizantes } \\
\hline & \multicolumn{3}{|c|}{$\mathrm{Y}$} & \multicolumn{3}{|c|}{$\mathrm{X}$} \\
\hline & \multicolumn{6}{|c|}{ Largura de Trabalho (m) para o Percurso } \\
\hline & \multirow{2}{*}{ Contínuo } & \multicolumn{2}{|c|}{ Alternado } & \multirow{2}{*}{ Contínuo } & \multicolumn{2}{|c|}{ Alternado } \\
\hline & & Esquerdo & Direito & & Esquerdo & Direito \\
\hline $\mathrm{N}$ & 7,3 & 7,8 & 7,0 & 8,0 & 8,1 & 7,5 \\
\hline $\mathrm{P}_{2} \mathrm{O}_{5}$ & 8,2 & 8,9 & 8,0 & 8,1 & 8,0 & 4,7 \\
\hline $\mathrm{K}_{2} \mathrm{O}$ & 6,8 & 7,4 & 4,3 & 7,7 & 7,9 & 4,6 \\
\hline $\mathrm{Ca}$ & 8,6 & 9,8 & 7,1 & 7,5 & 7,9 & 4,6 \\
\hline$S$ & 7,5 & 8,1 & 7,1 & 7,7 & 7,9 & 4,7 \\
\hline $\mathrm{Zn}$ & -- & -- & -- & 4,4 & 8,4 & 5,2 \\
\hline $\mathrm{B}$ & -- & -- & -- & 4,7 & 8,2 & 4,7 \\
\hline
\end{tabular}

Para proceder a análise da segregação com base nesses dados, foram comparadas as diferenças das larguras efetivas obtidas pelo ensaio da máquina para cada fertilizante estudado com aquelas relacionadas à largura calculada para a deposição de cada nutriente separadamente. Na Tabela 4, os dados representam o desvio da largura de cada nutriente para ambos os fertilizantes estudados, o que significa que, quanto menores esses desvios, menos segregação ocorre entre os nutrientes do fertilizante estudado, pois em uma distribuição ótima, sem nenhuma segregação, não haveria desvios nas larguras calculadas para cada nutriente individualmente, comparadas àquela inferida para o fertilizante.

TABELA 4. Diferenças das larguras efetivas de aplicação para cada fertilizante, utilizando C.V. de $15 \%$ na regularidade de distribuição transversal, em relação às larguras efetivas calculadas com o mesmo C.V., para a regularidade de distribuição de cada nutriente desses fertilizantes considerados individualmente. Effective width application differences for each fertilizer using a C.V. of $15 \%$ on the transversal regularity distribution in relation to the effective widths calculated with the same C.V. for the distribution regularity of each nutrient considered individually.

\begin{tabular}{|c|c|c|c|c|c|c|}
\hline \multirow{5}{*}{ Nutrientes } & \multicolumn{6}{|c|}{ Fertilizantes } \\
\hline & & $\mathrm{Y}$ & & & $\mathrm{X}$ & \\
\hline & \multicolumn{6}{|c|}{$\begin{array}{l}\text { Desvio da Largura de Trabalho (m) em Relação à Largura Efetiva de Aplicação do } \\
\text { Fertilizante para o Percurso }\end{array}$} \\
\hline & \multirow{2}{*}{ Contínuo } & \multicolumn{2}{|c|}{ Alternado } & \multirow{2}{*}{ Contínuo } & \multicolumn{2}{|c|}{ Alternado } \\
\hline & & Esquerdo & Direito & & Esquerdo & Direito \\
\hline $\mathrm{N}$ & 0,4 & 0,4 & 0,3 & $-0,1$ & $-0,1$ & 0,0 \\
\hline $\mathrm{P}_{2} \mathrm{O}_{5}$ & $-0,5$ & $-0,7$ & $-0,7$ & $-0,2$ & 0,0 & 2,8 \\
\hline $\mathrm{K}_{2} \mathrm{O}$ & 0,9 & 0,8 & 3,0 & 0,2 & 0,1 & 2,9 \\
\hline $\mathrm{Ca}$ & $-0,9$ & $-1,6$ & 0,2 & 0,4 & 0,1 & 2,9 \\
\hline $\mathrm{S}$ & 0,2 & 0,1 & 0,2 & 0,2 & 0,1 & 2,8 \\
\hline $\mathrm{Zn}$ & -- & -- & -- & 3,5 & $-0,4$ & 2,3 \\
\hline $\mathrm{B}$ & -- & -- & -- & 3,2 & $-0,2$ & 2,8 \\
\hline
\end{tabular}

Nesse caso, o fertilizante $\mathrm{X}$ apresentou maior segregação. A diferença foi maior no percurso alternado direito e para os nutrientes $\mathrm{P}_{2} \mathrm{O}_{5}, \mathrm{~K}_{2} \mathrm{O}$, Ca e S. Na Figura 4, ilustra-se a relação entre a largura efetiva e o C.V. para alguns nutrientes componentes do fertilizante $\mathrm{X}$ para o percurso 
contínuo. Nesse caso, observou-se que existe inflexão nas curvas do C.V. correspondente à largura de aplicação efetiva em torno de 7,5 m. A inflexão que mais se afastou do valor de C.V. de $15 \%$ foi aquela relacionada ao nutriente $\mathrm{Ca}$, com valor mínimo na inflexão de $17,4 \%$, e a que mais se aproximou desse índice foi a relacionada ao nutriente $\mathrm{K}_{2} \mathrm{O}$, com valor de $15,7 \%$.

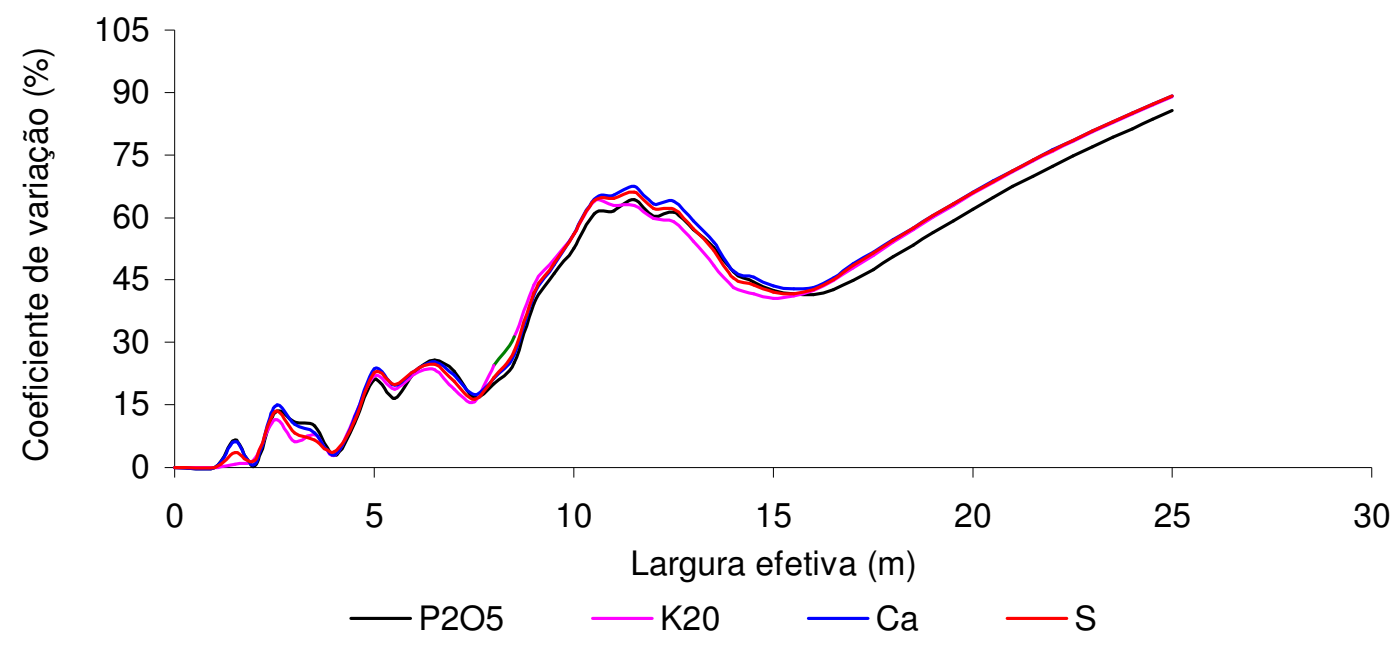

FIGURA 4. Relação entre o C.V. e largura de aplicação para os nutrientes do fertilizante X para o percurso contínuo. Relationship between C.V. and application width for the fertilizer $\mathrm{X}$ and continuous track.

Para identificar localizadamente a possível ocorrência de segregação dos fertilizantes, quantificou-se a formulação adquirida nos dois extremos e no centro da largura efetiva de aplicação de cada fertilizante, sendo os resultados ilustrados na Tabela 5 e válidos para o sistema de percurso contínuo.

TABELA 5. Formulação dos fertilizantes em pontos localizados da extensão da faixa de aplicação, válida para o sistema de percurso contínuo. Fertilizers formulas on specific locations along the transversal application profile for the continuous track.

\begin{tabular}{|c|c|c|c|c|c|c|c|c|}
\hline \multirow{3}{*}{ Nutrientes } & \multicolumn{8}{|c|}{ Fertilizantes } \\
\hline & \multicolumn{4}{|c|}{$\mathrm{Y}$} & \multicolumn{4}{|c|}{$\mathrm{X}$} \\
\hline & $\begin{array}{l}\text { Fórmula } \\
\text { Original }\end{array}$ & $\begin{array}{c}\text { Extremidade } \\
\text { Esquerda }\end{array}$ & Meio & $\begin{array}{c}\text { Extremidade } \\
\text { Direita }\end{array}$ & $\begin{array}{l}\text { Fórmula } \\
\text { Original }\end{array}$ & $\begin{array}{c}\text { Extremidade } \\
\text { Esquerda }\end{array}$ & Meio & $\begin{array}{c}\text { Extremidade } \\
\text { Direita }\end{array}$ \\
\hline $\mathrm{N}$ & 6,0 & 4,93 & 4,96 & 5,03 & 6,0 & 5,45 & 5,44 & 5,43 \\
\hline $\mathrm{P}_{2} \mathrm{O}_{5}$ & 18,0 & 16,65 & 16,96 & 16,60 & 18,0 & 17,18 & 17,08 & 16,99 \\
\hline $\mathrm{K}_{2} \mathrm{O}$ & 18,0 & 23,83 & 23,71 & 24,15 & 18,0 & 18,87 & 19,00 & 19,13 \\
\hline $\mathrm{Ca}$ & 5,0 & 5,67 & 5,90 & 5,55 & 5,0 & 6,95 & 6,82 & 6,68 \\
\hline $\mathrm{S}$ & 6,0 & 6,00 & 6,14 & 5,93 & 5,0 & 5,95 & 5,89 & 5,83 \\
\hline $\mathrm{Zn}$ & - & - & - & - & 0,10 & 0,19 & 0,19 & 0,20 \\
\hline $\mathrm{B}$ & - & - & - & - & 0,04 & 0,07 & 0,06 & 0,06 \\
\hline
\end{tabular}

Os resultados evidenciam que ambos os fertilizantes apresentaram diferenças na sua formulação em relação à posição da faixa de aplicação onde foram depositados. Essas diferenças são mais bem visualizadas por meio de valor numérico de desvio da fórmula original, ou seja, quanto esse valor difere percentualmente em relação à fórmula original, de forma semelhante ao que POPP \& ULRICCH, (1985) obtiveram com a fórmula 15-15-15. Na Tabela 6, mostra-se que realmente houve diferenças, ou seja, desvios em relação à fórmula original para cada um dos nutrientes estudados, os quais são numericamente maiores para o fertilizante $\mathrm{X}$, especialmente para os macronutrientes secundários Ca e S, e para os micronutrientes Zn e B. 
TABELA 6. Diferença percentual da formulação obtida nos dois extremos e no centro da largura efetiva da máquina em relação à fórmula original da mistura de grânulos aplicada. Percent differences on the fertilizer formula on the extremes and center of the machine effective width in relation to the original formula of the applied fertilizer.

\begin{tabular}{ccccccc}
\hline & \multicolumn{7}{c}{ Fertilizantes } \\
\cline { 2 - 6 } Nutrientes & \multicolumn{7}{c}{ Y } & \multicolumn{3}{c}{$\mathrm{X}$} \\
\cline { 2 - 7 } & \multicolumn{2}{c}{ Diferença em Relação ao Nutriente da Fórmula do Fertilizante (\%) } \\
\cline { 2 - 7 } & $\begin{array}{c}\text { Extremidade } \\
\text { Esquerda }\end{array}$ & Meio & $\begin{array}{c}\text { Extremidade } \\
\text { Direita }\end{array}$ & $\begin{array}{c}\text { Extremidade } \\
\text { Esquerda }\end{array}$ & Meio & $\begin{array}{c}\text { Extremidade } \\
\text { Direita }\end{array}$ \\
\hline $\mathrm{N}$ & $-17,8$ & $-17,3$ & $-16,1$ & $-9,2$ & $-9,3$ & $-9,5$ \\
$\mathrm{P}_{2} \mathrm{O}_{5}$ & $-7,5$ & $-5,7$ & $-7,7$ & $-4,6$ & $-5,1$ & $-5,6$ \\
$\mathrm{~K}_{2} \mathrm{O}$ & 32,3 & 31,7 & 34,1 & 4,8 & 5,6 & 6,2 \\
$\mathrm{Ca}$ & 13,4 & 18,0 & 11,0 & 39,0 & 36,4 & 33,6 \\
$\mathrm{~S}$ & 0,0 & 2,3 & $-1,1$ & 19,0 & 17,8 & 16,6 \\
$\mathrm{Zn}$ & -- & -- & -- & 90,0 & 90,0 & 100,0 \\
$\mathrm{~B}$ & -- & -- & -- & 75,0 & 50,0 & 50,0 \\
\hline
\end{tabular}

Observa-se, pelos resultados das larguras efetivas individuais de cada elemento (Tabela 3), que os valores para os micronutrientes foram baixos, o que também indica uma distribuição de pior qualidade destes. Contudo, é importante considerar que toda a análise é baseada apenas na formulação obtida nos coletores ao longo da faixa de aplicação. Não se considerou se essa formulação apresentava desvios no material original que foi utilizado nos ensaios de campo. Nesse caso, não seria efeito da máquina em segregar ou alterar a formulação ao aplicar o produto. Ao abordar questões relacionadas à qualidade da distribuição de fertilizantes, bem como aos conceitos de aplicação de fertilizantes em taxa variada, tais aspectos não são considerados.

A acurácia da aplicação de fertilizantes é parcialmente comprometida pela segregação, tanto maior quanto mais distante forem arremessadas as partículas, e atualmente estão disponíveis no mercado brasileiro distribuidores a lanço para larguras de trabalho de até $36 \mathrm{~m}$.

No presente caso, observaram-se diferenças consideráveis na aplicação de elementos individualmente, o que induz à busca de soluções como produtos de melhor qualidade e aqueles com mistura de elementos no próprio grão. No entanto, essa não é a solução para a aplicação em taxa variada, em que os elementos devem ser aplicados individualmente.

\section{CONCLUSÕES}

Ambos os fertilizantes estudados apresentaram segregação de seus nutrientes ou alteração na formulação com base naquela especificada quando aplicados com máquinas de distribuição centrífuga, como função da sua posição transversal em relação ao centro da máquina. No presente estudo, a maior alteração ocorreu no fertilizante $\mathrm{Y}$ para o nutriente $\mathrm{K}_{2} \mathrm{O}$ e no fertilizante $\mathrm{X}$ para os macronutrientes secundários $\mathrm{Ca}$ e $\mathrm{S}$ e para os micronutrientes $\mathrm{Zn}$ e $\mathrm{B}$.

\section{REFERÊNCIAS}

ALCARDE, J.C.; GUIDOLIN, J.A.; LOPES, A.S. Os adubos e a eficiência das adubações. São Paulo: ANDA, 1989. 35 p.

ASAE. AMERICAN SOCIETY OF AGRICULTURAL ENGINEERS. ASAE Standard: procedure for measuring distribution uniformity and calibrating granular broadcast spreaders. St. Joseph, 1995. 3 p.

GONÇALVES, A.O.; MOLIN, J.P.; MENEGATTI, L.A.A. Adulanço 2.0: software para análise de distribuição transversal. Revista Brasileira de Agrocomputação, Lavras, v.5, n.1, p.42-48, 2003. 
ISO. INTERNATIONAL ORGANIZATION FOR STANDARDIZATION. Equipment for distributing fertilizers: Test methods - Part 1: Full width fertilizer distributors. Géneve, 1985. 5 p. (ISO Standard 5690/1).

MALAVOLTA, E.; PEMENTEL-GOMES, F.; ALCARDE, J.C. Adubos e adubações. São Paulo: Nobel, 2000. 197 p.

MOHSENIN, N.N. Physical properties of plant and animal materials. $2^{\text {nd }}$ ed. New York: Gordon and Brechen Science Publ., 1986. 891 p.

MOLIN, J.P.; MAZZOTTI, H.C. Influência da utilização e do tipo de amortecedores de ricochete em ensaios de aplicadores a lanço. Revista Brasileira de Engenharia Agrícola e Ambiental, Campina Grande, v.4, n.2, p. 281-285, 2000.

POPP, T.; ULRICCH, K.H. Aplicación y calidad de los abonos complejos. BASF Reportes Agrícolas, Limburferhof, v.3, p.14-23, 1985.

SMITH, G.E. Bulk blended. In: ANNUAL MEETING OF THE FERTILIZER INDUSTRY

ROUND TABLE, 11., 1960, Washington. Proceedings... Maumee: Andersons; 1960. v.9, p.90-97. 